\title{
An Approach to Breast Cancer Diagnosis via PET Imaging of Microcalcifications Using ${ }^{18}$ F-NaF
}

\author{
George H. Wilson III ${ }^{1}$, John C. Gore ${ }^{1-4}$, Thomas E. Yankeelov ${ }^{1-3,5}$, Stephanie Barnes ${ }^{1,2}$, Todd E. Peterson ${ }^{1,2}$, \\ Jarrod M. True ${ }^{1}$, Sepideh Shokouhi ${ }^{1,2}$, J. Oliver McIntyre ${ }^{1-3,5}$, Melinda Sanders ${ }^{6}$, Vandana Abramson ${ }^{7}$, \\ The-Quyen Ngyuen ${ }^{3}$, Anita Mahadevan-Jansen ${ }^{3}$, and Mohammed N. Tantawy ${ }^{1,2}$ \\ ${ }^{1}$ Vanderbilt University Institute of Imaging Science, Vanderbilt University Medical Center, Nashville, Tennessee; ${ }^{2}$ Department of \\ Radiology and Radiological Sciences, Vanderbilt University Medical Center, Nashville, Tennessee; ${ }^{3}$ Department of Biomedical \\ Engineering, Vanderbilt University Medical Center, Nashville, Tennessee; ${ }^{4}$ Department of Molecular Physiology and Biophysics, \\ Vanderbilt University Medical Center, Nashville, Tennessee; ${ }^{5}$ Department of Cancer Biology, Vanderbilt University Medical Center, \\ Nashville, Tennessee; ${ }^{6}$ Department of Pathology, Vanderbilt University Medical Center, Nashville, Tennessee; and ${ }^{7}$ Department of \\ Hematology/Oncology, Vanderbilt University Medical Center, Nashville, Tennessee
}

Current radiologic methods for diagnosing breast cancer detect specific morphologic features of solid tumors or any associated calcium deposits. These deposits originate from an early molecular microcalcification process of 2 types: type 1 is calcium oxylate and type II is carbonated calcium hydroxyapatite. Type I microcalcifications are associated mainly with benign tumors, whereas type II microcalcifications are produced internally by malignant cells. No current noninvasive in vivo techniques are available for detecting intratumoral microcalcifications. Such a technique would have a significant impact on breast cancer diagnosis and prognosis in preclinical and clinical settings. ${ }^{18} \mathrm{~F}-\mathrm{NaF}$ PET has been used solely for bone imaging by targeting the bone hydroxyapatite. In this work, we provide preliminary evidence that ${ }^{18} \mathrm{~F}-\mathrm{NaF}$ PET imaging can be used to detect breast cancer by targeting the hydroxyapatite lattice within the tumor microenvironment with high specificity and softtissue contrast-to-background ratio while delineating tumors from inflammation. Methods: Mice were injected with approximately $10^{6}$ MDA-MB-231 cells subcutaneously and imaged with ${ }^{18} \mathrm{~F}-\mathrm{NaF}$ PET/ CT in a 120-min dynamic sequence when the tumors reached a size of $200-400 \mathrm{~mm}^{3}$. Regions of interest were drawn around the tumor, muscle, and bone. The concentrations of radiotracer within those regions of interest were compared with one another. For comparison to inflammation, rats with inflamed paws were subjected to ${ }^{18} \mathrm{~F}-$ NaF PET imaging. Results: Tumor uptake of ${ }^{18} \mathrm{~F}^{-}$was significantly higher $(P<0.05)$ than muscle uptake, with the tumor-to-muscle ratio being about 3.5. The presence of type II microcalcification in the MDA-MB-231 cell line was confirmed histologically using alizarin red $\mathrm{S}$ and von Kossa staining as well as Raman microspectroscopy. No uptake of ${ }^{18} \mathrm{~F}^{-}$was observed in the inflamed tissue of the rats. Lack of hydroxyapatite in the inflamed tissue was verified histologically. Conclusion: This study provides preliminary evidence suggesting that specific targeting with ${ }^{18} \mathrm{~F}^{-}$of hydroxyapatite within the tumor microenvironment may be able to distinguish between inflammation and cancer.

Received Feb. 20, 2014; revision accepted Apr. 2, 2014.

For correspondence or reprints contact: Mohammed N. Tantawy, Vanderbilt University, 1161 21st Ave. S., MCN AA-1105, Nashville, TN 37232.

E-mail: n.tantawy@vanderbilt.edu

Guest editor: Johannes Czernin, UCLA School of Medicine

Published online May 15, 2014.

COPYRIGHT (c) 2014 by the Society of Nuclear Medicine and Molecular Imaging, Inc.
Key Words: breast cancer; MDA-MB-231; microcalcification; ${ }^{18} \mathrm{~F}-\mathrm{NaF}$; ${ }^{18} \mathrm{~F}^{-}$; PET; hydroxyapatite; calcium oxalate

J Nucl Med 2014; 55:1138-1143

DOI: 10.2967/jnumed.114.139170

B United States. It is the second leading cause of cancer death among women, causing almost 40,000 deaths in 2011 alone (1). Current imaging techniques may fail to detect tumors (false-negative) because of their small size or location in the breast, obscuration by nearby organs, or dense breast tissue (2).

One significant feature of breast cancer diagnosis is the presence of calcium deposits (averaging $0.3 \mathrm{~mm}^{3}$ in size) detected via mammograms (3-5). These calcium deposits are potentially the result of condensation of 1 of 2 types of microcalcification found within the tumor microenvironment: type I, which contains calcium oxalate dehydrate, and type II, which contains calcium phosphates in the form of hydroxyapatite (6). Importantly, type I deposits are associated with benign breast disease, whereas malignant cells have the unique capability of producing hydroxyapatite $(4,7,8)$. Alkaline phosphatase on the surface of malignant cells hydrolyzes $\beta$-glycerophosphate to glycerol and inorganic phosphate, which is transported into the cell by the type II family of sodium-inorganic phosphate cotransporters. There, the inorganic phosphate combines with calcium to produce hydroxyapatite crystals. Hydroxyapatite then leaves the cells, by unknown mechanisms, to enter the extracellular matrix. Furthermore, hydroxyapatite enhances the mitogenesis of mammary cells, amplifying the malignant process and resulting in accelerated tumor growth $(7,8)$. Therefore, hydroxyapatite may be a biomarker for breast malignancy.

Apatite calcification in bone is generally composed of hydroxyapatite (9). The carbonate substitution for phosphate in the bioapatites significantly increases the reactivity of these compounds, especially to anions such as fluoride, allowing them to substitute into the lattice (10). Sodium fluoride labeled with ${ }^{18} \mathrm{~F}^{-}\left({ }^{18} \mathrm{~F}-\mathrm{NaF}\right)$ 


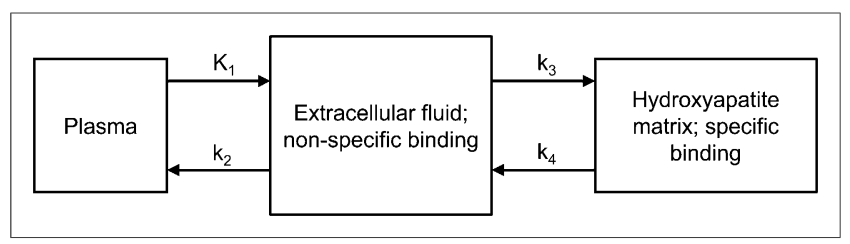

FIGURE 1. A 3-compartment model was used to describe kinetic distribution of ${ }^{18} \mathrm{~F}^{-}$ions to tumor and bone. $K_{1}$ is transfer rate of ${ }^{18} \mathrm{~F}^{-}$from compartment 1 (plasma) to compartment 2 (extracellular fluid space) in units of $\mathrm{mL} / \mathrm{min} / \mathrm{g}, k_{2}$ is transfer rate from 2 to 1 in units of $1 / \mathrm{min}, k_{3}$ is transfer rate from 2 to 3 (hydroxyapatite lattice) in units of $1 / \mathrm{min}$, and $k_{4}$ is transfer rate from 3 to 2 in units of $1 / \mathrm{min}$.

has previously been used for bone imaging and bone hydroxyapatite abundance quantification, as well as for detecting bone metastases using PET. The free fluoride dissociates from the sodium and binds to the hydroxyapatite matrix $\left(\mathrm{Ca}_{10}\left(\mathrm{PO}_{4}\right)_{6} \mathrm{OH}_{2}\right)$ of the skeleton (11), where ${ }^{18} \mathrm{~F}^{-}$substitutes for the $\mathrm{OH}^{-}$of the hydroxyapatite and forms fluoroapatite $\left(\mathrm{Ca}_{10}\left(\mathrm{PO}_{4}\right)_{6} \mathrm{~F}_{2}\right)(12)$.

Our working hypothesis is that the same mechanisms of uptake of ${ }^{18} \mathrm{~F}^{-}$in bone apply to breast tumors containing hydroxyapatite within their microenvironment. Therefore, we investigated the ability of ${ }^{18} \mathrm{~F}-\mathrm{NaF}$ to detect breast tumors via targeting of the hydroxyapatite microenvironment using mouse models of MDAMB-231, a triple-negative human breast cancer cell line that does not express the genes for estrogen receptor, progesterone receptor, or Her2/neu. MDA-MB-231 cells produce highly invasive malignant tumors (13). Thus, this cell line is a prototype for highly differentiated breast cancer cells with overexpressed epidermal growth factor receptors (14). We then assessed the ability of this technique to discriminate between inflammation and cancer by applying it to a rat model of acute inflammation.

\section{MATERIALS AND METHODS}

All studies were approved by the Vanderbilt University Animal Care and Use Committee before the experiments began.

\section{Tumor Model and Imaging}

MDA-MB-231 cells were cultured in Dulbecco modified Eagle medium (Invitrogen) supplemented with $10 \%$ fetal bovine serum and $1 \%$ penicillin streptomycin (Invitrogen) at $37^{\circ} \mathrm{C}$ in a humidified, $5 \%$ $\mathrm{CO}_{2}$ incubator. Four- to 5-wk-old female Foxn1 nu/nu mice were injected subcutaneously in the right hind limb $(n=10)$ with about 10 million MDA-MB-231 cells in a 1:2 ratio of Matrigel and Dulbecco modified Eagle medium.

Once the tumors reached $200-400 \mathrm{~mm}^{3}$, the mice were imaged in a microPET Focus 220 (Siemens Preclinical Solutions) for $120 \mathrm{~min}$ in dynamic acquisition mode with a simultaneous intravenous injection of about $18 \mathrm{MBq}$ of ${ }^{18} \mathrm{~F}-\mathrm{NaF}$, provided by the Vanderbilt Radiochemistry Core. All mice were anesthetized with $2 \%$ isoflurane vaporized with oxygen at a steady flow rate of $2.5 \mathrm{~L} / \mathrm{min}$ during imaging. The dynamic acquisitions consisted of twelve 5-s frames, four 60-s frames, one 5-min frame, and eleven 10-min frames. All datasets were reconstructed using the maximum a posteriori algorithm into $128 \times 128 \times 95$ slices with a voxel size of $0.95 \times 0.95 \times$ $0.8 \mathrm{~mm}$ at a $\beta$ value of 0.001 . Immediately after the PET scans, the mice were imaged in a microCAT II (Siemens) at an $\mathrm{x}$-ray beam intensity of $180 \mathrm{mAs}$ and an $\mathrm{x}$-ray tube voltage of $80 \mathrm{kVp}$ for anatomic coregistration with the PET images. The images were reconstructed in a $512 \times 512 \times 512$ matrix with a voxel size of $0.122 \times$ $0.122 \times 0.173 \mathrm{~mm}$.

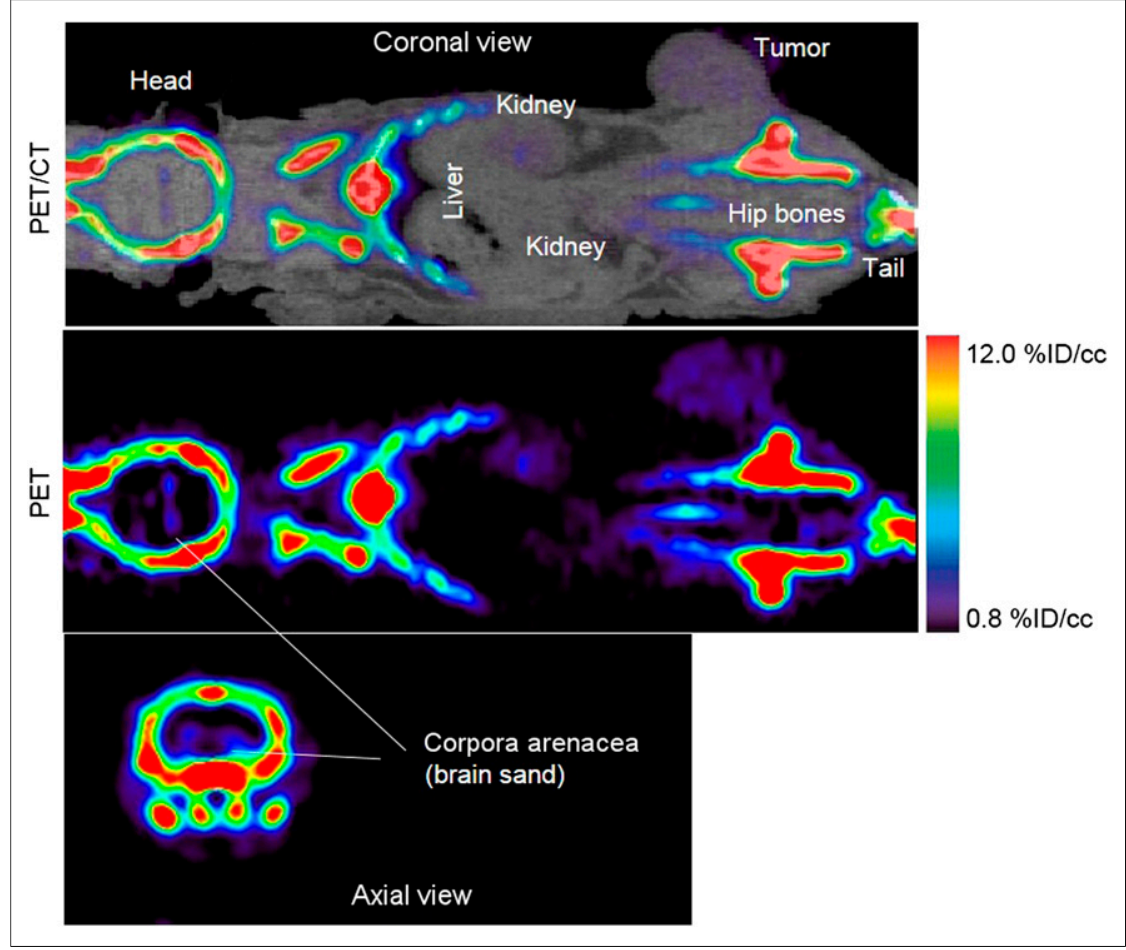

FIGURE 2. A 60 - to $80-$ min summed PET/CT and PET image of ${ }^{18} \mathrm{~F}^{-}$uptake in mouse bearing MDA-MB-231 tumor and imaged for $120 \mathrm{~min}$. With windowing level set between 0.8 and $12 \% \mathrm{ID} / \mathrm{cc}$, bone image is saturated but tumor and brain sand are visible with high contrast-to-background softtissue ratio

\section{Inflammation Model and Imaging}

Carrageenan $(100 \mu \mathrm{L}$ of a $1 \%$ solution in sterile saline) was injected subcutaneously into the rear right footpad of wild-type Sprague Dawley rats $(n=3)$. Two hours later, when inflammation was maximal (15), the rats were injected with ${ }^{18} \mathrm{~F}-\mathrm{NaF}$ and imaged in our microPET Focus 220 for $30 \mathrm{~min}$ at $1 \mathrm{~h}$ after radiotracer administration in static mode followed by a CT scan as described earlier.

\section{Histologic Search for Hydroxyapatite}

Staining. Immediately after PET/CT imaging, the tumors and muscle were harvested. For the rat inflammatory model, the feet were harvested at the end of the imaging session. Sections of the harvested tissue $(5 \mu \mathrm{m})$ were loaded onto glass slides and assessed for mineralization via alizarin red $\mathrm{S}$ and von Kossa staining. The staining process was performed at the Vanderbilt Translational Pathology Shared Resource, which uses manufacturerprovided staining protocols. Positive staining for alizarin red $\mathrm{S}$ (color, red) indicates the presence of calcium, whereas positive staining for von Kossa (color, black/brown) indicates the presence of calcium phosphates (8).

Raman Microspectroscopy. The stained tissue sections, along with stained mouse bone sections provided by the Translational Pathology Core at Vanderbilt University, were subjected to Raman microspectroscopy using 


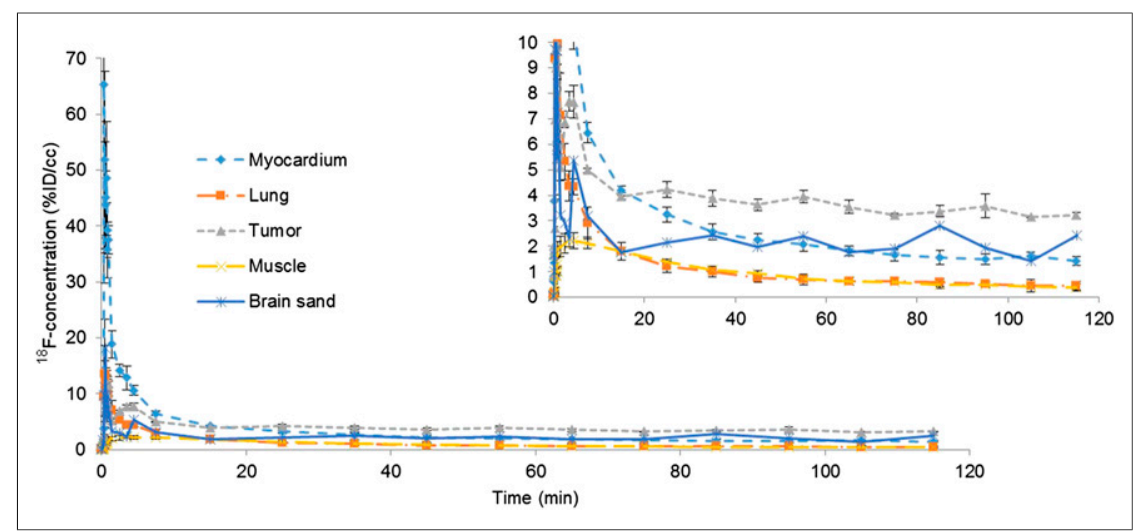

FIGURE 3. Time-activity curves of different regions in mouse injected with ${ }^{18} \mathrm{~F}-\mathrm{NaF}$ displayed in Figure 2. \%ID/cc is ${ }^{18} \mathrm{~F}^{-}$concentration within each ROI normalized to total injected dose. Insert is zoomed view of time-activity curves.

an inVia Raman microscope (Renishaw) with a 785-nm laser and a $\times 50$ objective (numeric aperture, 0.75 ). Regions of $100 \times 60 \mu \mathrm{m}$ of the sample were mapped over the entire section, and Raman spectra were acquired over those areas with an axial resolution of $1 \mu \mathrm{m}$. A shift near $960 \mathrm{~cm}^{-1}$ is typical of hydroxyapatite $(6,8,16)$. Spectra were processed using WiRE software, version 3.3 (Renishaw). The bone slides were used for initial calibration. On the basis of the Raman spectra, regions on the mapped sample were classified using a direct classic least-squares method and were color-coded in green to indicate a $960 \mathrm{~cm}^{-1}$ shift or black to indicate no shift.

\section{Data Analysis}

Anatomic regions of interest (ROIs) were drawn around the entire tumor, hip bones, lungs, heart, and muscle of the opposing hind limb on the CT images for each mouse using the medical imaging analysis tool AMIDE (17) and superimposed on the PET images. In addition, ROIs were drawn around the corporal arenacea (brain sand) directly on the PET images because CT cannot accurately distinguish brain regions. Time-activity curves were established for all ROIs over the entire duration of the dynamic scans. For the rat inflammation studies, ROIs were drawn around each paw on the CT images and superimposed on the PET images.

The distribution of ${ }^{18} \mathrm{~F}^{-}$is determined by blood flow initially and then clears rapidly from plasma and is excreted by the kidneys (12). In the bone, the ${ }^{18} \mathrm{~F}^{-}$ions pass through plasma to the extracellular fluid space, into the shell of bound water on the hydroxyapatite crystal surface, and into the interior of the crystal, where ${ }^{18} \mathrm{~F}^{-}$exchanges for $\mathrm{OH}^{-}$ on the hydroxyapatite lattice. The kinetics of ${ }^{18} \mathrm{~F}^{-}$moving from the blood plasma to incorporation into fluoroapatite have been described by a 3-compartment model $(12,18)$ as demonstrated in Figure 1. Therefore, we used a similar model to describe tumor uptake of ${ }^{18} \mathrm{~F}^{-}$.

The mean concentrations of ${ }^{18} \mathrm{~F}^{-}$within a 20 -min interval between 40 and 60 min after ${ }^{18} \mathrm{~F}-\mathrm{NaF}$ injection were normalized to the total injected dose $(\% \mathrm{ID} / \mathrm{cc})$ for the tumor, muscle, and bone regions of the mice. This time window was chosen on the basis of the results described below. Similarly, the \%ID/cc for the rat paws was measured for the entire scan (30 min). A 2-tailed Student $t$ test was used to compare \% ID/cc between tumor and muscle in rats and between tumor and bone in mice; the statistical significance threshold was considered to be at a $P$ value of 0.05 .

\section{RESULTS}

Initial perfusion of ${ }^{18} \mathrm{~F}^{-}$was observed in all tissues, including tumor and muscle, within the first minute after injection of ${ }^{18} \mathrm{~F}-\mathrm{NaF}$. The ${ }^{18} \mathrm{~F}^{-}$ions were quickly excreted by the kidneys, and by about $40 \mathrm{~min}$, residual ions were concentrated in the bone and tumor with little or no signal observed in the muscle, as shown in Figure 2. The time-activity curves were fairly flat from 40 to $120 \mathrm{~min}$, indicating a state of equilibrium, as demonstrated in Figure 3. Washout of the radiotracer from bone and tumor was fairly slow as indicated by a negligible $k_{4}$ (Table 1 ). Therefore, we measured the ${ }^{18} \mathrm{~F}^{-}$uptake constant as $K_{\mathrm{1s}_{\mathrm{F}}}=K_{1} k_{3} /\left(k_{2}+k_{3}\right)$.

The concentration of ${ }^{18} \mathrm{~F}^{-}$in the tumor between 60 and $80 \mathrm{~min}$ was about 3.5 times higher than that of muscle $(n=7, P<0.05)$, where the $\% \mathrm{ID} / \mathrm{cc}$ within the tumor ROI was $2.9 \pm 0.8$ (mean \pm SD) and those of muscle and bone were $0.8 \pm 0.2$ and $31 \pm 4$, respectively.

Positive staining for calcium and calcium phosphate was observed in both tumor and bone, as shown in Figure 4. The positive von Kossa staining suggests the presence of type II calcifications specifically (8). A Raman shift of about $960 \mathrm{~cm}^{-1}$ was observed for the MDA-MB-231 slides as shown in Figure 4, where the green color over the tumor section in the $100 \times 60 \mu \mathrm{m}^{2}$ Raman image indicates that a shift near $960 \mathrm{~cm}^{-1}$ was found within that region. Thus, we confirmed the presence of carbonated calcium hydroxyapatite, type II microcalcifications, in the MDA-MB-231 microenvironment. No positive staining using alizarin red $\mathrm{S}$ or von Kossa was observed for the muscle, nor did we observe a hydroxyapatite Raman shift in these tissues.

In some cases, we observed a localized region that had a high concentration of ${ }^{18} \mathrm{~F}^{-}(\sim 9$ times higher than muscle, $\% \mathrm{ID} / \mathrm{cc}>8.5$, $n=3$ ), as shown in Figure 5. Interestingly, these mice were also the only mice with tumors that had started to ulcerate. In addition, no solid calcium deposits were observed in the CT scans. These mice were excluded from the statistical analysis since their $\% \mathrm{ID} / \mathrm{cc}$ values were more than twice the SD from the mean (physiologically, the skin surrounding the tumor was red, indicating the initial stages of

TABLE 1

Kinetic Parameters of ${ }^{18} \mathrm{~F}^{-}$Uptake in Mice Bearing MDA-MB-231 Tumors (Tumors Contain Hydroxyapatite Similar to What Is Found in Normal Bone)

\begin{tabular}{lccccc}
\hline Region & $K_{1}(\mathrm{~mL} / \mathrm{g} / \mathrm{min})$ & $k_{2}(1 / \mathrm{min})$ & $k_{3}(1 / \mathrm{min})$ & $k_{4}(1 / \mathrm{min})$ & $K$ \\
\hline Tumor & $0.42 \pm 0.19$ & $1.27 \pm 0.92$ & $0.07 \pm 0.06$ & $0.03 \pm 0.02$ & $0.03 \pm 0.01$ \\
Bone & $0.25 \pm 0.11$ & $0.44 \pm 0.12$ & $0.60 \pm 0.42$ & $0.002 \pm 0.002$ & $0.14 \pm 0.08$
\end{tabular}

Data are mean \pm SD. $K^{18_{\mathrm{F}}}$ is ${ }^{18} \mathrm{~F}^{-}$uptake constant described as $K_{1} k_{2} /\left(k_{2}+k_{3}\right)$. 


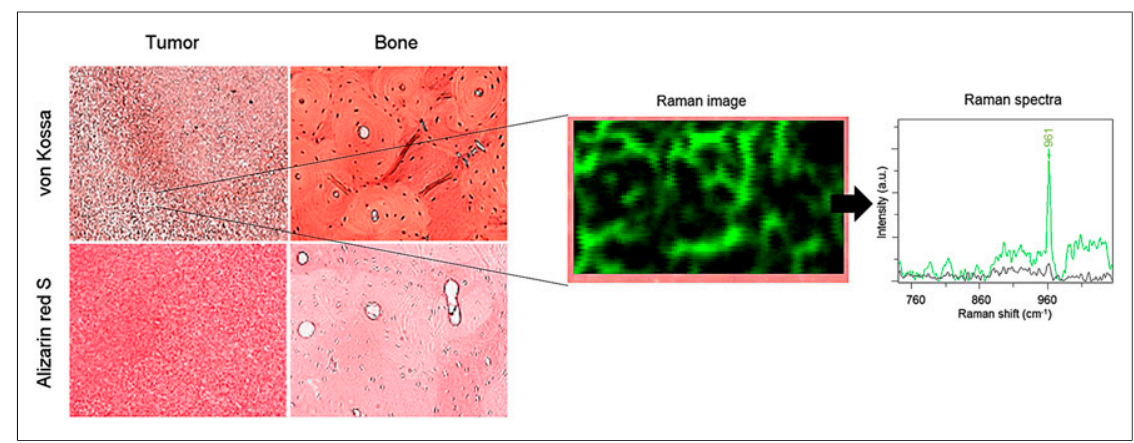

FIGURE 4. Staining of MDA-MB-231 breast cancer samples and normal bone with von Kossa (red/brown) and alizarin red S (red). Positive von Kossa staining indicate presence of calcium carbonates, suggesting presence of type II calcification specifically. A $100 \times 60 \mu \mathrm{m}^{2}$ region of same tumor section scanned with Raman microscope reveals locations within that region where $960 \mathrm{~cm}^{-1}$ shift was found (green color in Raman image and Raman spectra) and where there was no shift near $960 \mathrm{~cm}^{-1}$ (black color). Shift at $\sim 960 \mathrm{~cm}^{-1}$ indicates presence of hydroxyapatite. a.u. $=$ arbitrary units.

ulceration). Autoradiography correlated well with the von Kossa stain results, as shown in Figure 5.

We did not observe any ${ }^{18} \mathrm{~F}^{-}$uptake, or positive staining for alizarin red $\mathrm{S}$ or von Kossa, in the inflamed soft tissue of the rat paws, as shown in Figure 6; for comparison, \%ID/cc of noninflamed soft tissue and paw bone were $0.9 \pm 0.4$ and $29 \pm 3$, respectively. As expected, no Raman shift at about $960 \mathrm{~cm}^{-1}$ was detected in the inflamed tissue, indicating the lack of hydroxyapatite in it (data not shown because the Raman images appear as just black rectangles).

Accumulation of ${ }^{18} \mathrm{~F}^{-}$was also observed in the corpora arenacea (brain sand), known to contain hydroxyapatite in humans and animals (19-21), as shown in Figure 2.

\section{DISCUSSION}

This study presents the first - to our knowledge-proposed use of ${ }^{18} \mathrm{~F}-\mathrm{NaF}$ PET imaging to specifically detect tumors on the basis of their hydroxyapatite abundance. The ${ }^{18} \mathrm{~F}^{-}$concentration was approximately 10 times higher in bone than in tumors, as is

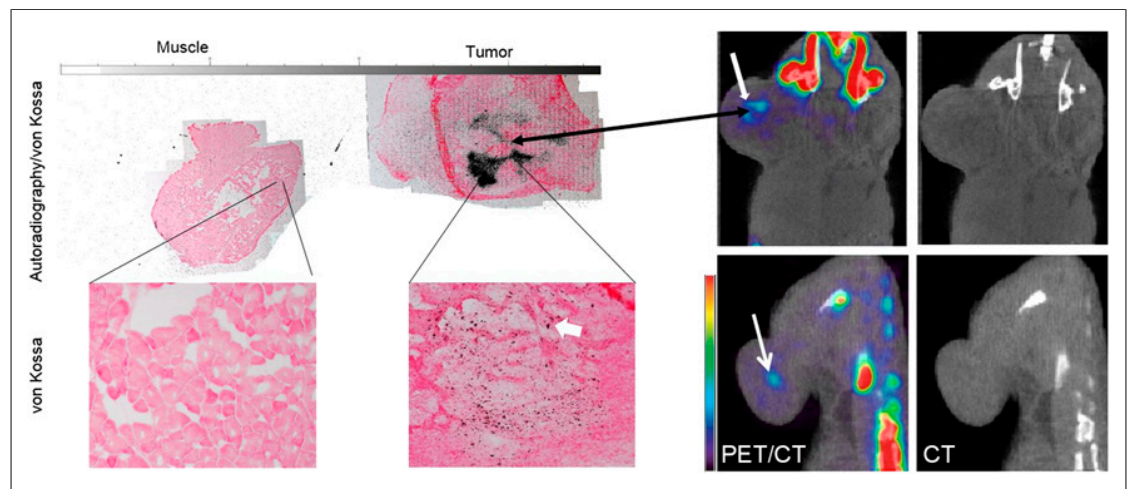

FIGURE 5. Coronal view of PET/CT and CT-only images of 2 mice injected with ${ }^{18} \mathrm{~F}-\mathrm{NaF}$ (right) and corresponding autoradiography/von Kossa staining of tumor section and normal muscle of one of the mice (left). Thick white arrow in stained image points to red/brown spot representing positive von Kossa staining. Localized region, indicated by white arrows in PET/CT images, with high ${ }^{18} \mathrm{~F}^{-}$concentration (about 9 times higher than muscle and 3 times lower than bone) were observed in tumors in PET images, indicating dense hydroxyapatite. These regions were not observed in CT images. Autoradiography correlated well with PET and staining results. expected because of the high density of hydroxyapatite in the bone and the large bone surface area. This higher concentration of ${ }^{18} \mathrm{~F}^{-}$in bone than in tumors was also indicated by $K_{{ }_{\mathrm{F}}}$ (Table 1). Therefore, to visually identify the tumor in the PET images, the windowing level was adjusted to a maximum of $12 \% \mathrm{ID} / \mathrm{cc}$, as shown in Figure 2, causing the bone signal to appear saturated. For bone structure identification, the high end of the scale bar would be set to at least $45 \%$ so that the tumor is not visible. In addition, the minimum windowing level was set to $0.8 \% \mathrm{ID} / \mathrm{cc}$ to mask normal tissue perfusion (e.g., muscle). All analyses of PET images in this work used these same windowing limits.

Of the limited number of studies performed on hydroxyapatite and breast cancer, we are not aware of any that included negative control tumor models. Because breast lesions may be inflammatory lesions, we used an inflammatory rodent model to study the ability of ${ }^{18} \mathrm{~F}^{-}$PET images to distinguish between inflammation and malignancy. No accumulation of ${ }^{18} \mathrm{~F}^{-}$occurred in the rat inflammatory tissue because of lack of hydroxyapatite (Fig. 6). Therefore, this technique may indeed represent a novel and potentially reliable noninvasive in vivo method for distinguishing between malignant and inflammatory lesions with high specificity or between lesions that contain hydroxyapatite and those that do not. No other imaging modalities and techniques have been shown to have this capability. In addition, this technique can be used to measure hydroxyapatite abundance and the relationship between tumor progression and hydroxyapatite in preclinical settings.

The lack of accumulation of ${ }^{18} \mathrm{~F}^{-}$in the normal soft tissue, taken with the accumulation of ${ }^{18} \mathrm{~F}^{-}$in the brain sand and the tumor, supports the specificity of ${ }^{18} \mathrm{~F}^{-}$in binding to hydroxyapatite when present in soft tissue. This binding occurs through mechanisms similar to those of bone uptake of ${ }^{18} \mathrm{~F}^{-}$, with a high softtissue contrast-to-background ratio because the tracer does not accumulate in soft tissue not containing hydroxyapatite.

Calcium oxalate is implicated in the composition of most kidney stones (22$25)$. Prolonged exposure to high concentrations of $\mathrm{NaF}(60 \mathrm{mM})$ has been shown to be able to dissolve calcium oxalate in vitro, but this concentration would be considered toxic in vivo (26). Since radiotracer concentrations, including ${ }^{18} \mathrm{~F}-\mathrm{NaF}$, are on the order of submicromolar or smaller (27), it is highly unlikely that ${ }^{18} \mathrm{~F}-\mathrm{NaF}$ can break the calcium oxalate bond in benign breast lesions. Therefore, this technique can be used not only to detect single and multicentric tumors with high contrast-to-background ratio but also to differentiate between type I and type II microcalcifications within the tumor's microenvironment.

This imaging technique can rapidly be translated to clinical trials since ${ }^{18} \mathrm{~F}$ $\mathrm{NaF}$, or ${ }^{18} \mathrm{~F}^{-}$, is already Food and Drug 




FIGURE 6. (Left) Rat model of acute inflammation (in right footpad) imaged with ${ }^{18} \mathrm{~F}-\mathrm{NaF}$ PET at maximal inflammation. Uptake of ${ }^{18} \mathrm{~F}^{-}$was limited to bone, with no accumulation in inflammatory soft tissue because of lack of hydroxyapatite. (Right) Section of inflamed tissue stained with von Kossa. No positive staining (or red/brown spots) was observed within stained tissue, indicating no presence of calcium phosphates or type II microcalcification. Lower and upper limits on scale bar are 0.8 and $12 \% \mathrm{ID} / \mathrm{cc}$. breast cancer cells progressing from chronic inflammation, ductal carcinoma in situ, or lobular carcinoma in situ, avoiding repeated biopsies.

\section{DISCLOSURE}

The costs of publication of this article were defrayed in part by the payment of page charges. Therefore, and solely to indicate this fact, this article is hereby marked "advertisement" in accordance with 18 USC section 1734. The National Institutes of Health provided funding via NCI R01CA138599, NCI P30CA68485, and NCI P50CA098131, and the Kleberg Foundation provided generous support of the Imaging Program. No other potential conflict of interest relevant to this article was reported.

\section{ACKNOWLEDGMENT}

We thank Dr. Zou Yue for animal support.
Administration-approved. Although the approach is not appropriate for screening, it could be deployed to support mammograms when suggestive lesions or multicentric lesions are detected and biopsy is not feasible. The total-body adsorbed dose is about 0.024 $\mathrm{mSv} / \mathrm{MBq}(0.989 \mathrm{mrem} / \mathrm{mCi})$, or $4.44 \mathrm{mSv}$ for a typical $185-\mathrm{MBq}$ (5-mCi) dose of ${ }^{18} \mathrm{~F}-\mathrm{NaF}$ injection, which is well within the radiation dose tolerance for nonradiation workers (28). Additionally, because microcalcifications were detected with ${ }^{18} \mathrm{~F}-\mathrm{NaF}$ PET imaging when no solid calcium deposits were observed on the CT scan, this technique could be useful in early breast cancer diagnosis before solid calcium deposits are visible on mammograms. This ability would be especially important in cases of chronic inflammation, ductal carcinoma in situ, or lobular carcinoma in situ, which could progress to malignancy over time (29-31).

Previous studies on breast cancer have used ${ }^{18} \mathrm{~F}^{-}$and sometimes a cocktail of ${ }^{18} \mathrm{~F}^{-}$and ${ }^{18} \mathrm{~F}-\mathrm{FDG}(32,33)$. Those studies, however, focused on bone metastases of breast cancer, and the cancer was at an advanced stage and had already spread and metastasized to different locations of the skeleton. Our work suggests the use of ${ }^{18} \mathrm{~F}^{-}$imaging within the breast and at a very early stage for primary detection rather than metastasis detection.

\section{CONCLUSION}

The results from this study provide the first evidence, to our knowledge, that PET imaging of breast cancer with ${ }^{18} \mathrm{~F}^{-}$via specific targeting of hydroxyapatite within the tumor microenvironment may be able to sensitively and noninvasively identify intratumoral microcalcifications with high specificity and contrastto-background ratio, as this technique targets type II microcalcifications in vivo. Because of radiation and cost, this technique could not be used for initial screening for breast cancer. However, it can be used for identifying multicentric tumors, as well as for discriminating between malignancies and benign factors (e.g., inflammation, cysts, and fat necrosis). Additionally, this technique can be used for the early detection of

\section{REFERENCES}

1. DeSantis C, Siegel R, Bandi P, Jemal A. Breast cancer statistics, 2011. CA Cancer J Clin. 2011;61:409-418.

2. Bruening W, Uhl S, Fontanarosa J, Reston J, Treadwell J, Schoelles K. Noninvasive Diagnostic Tests for Breast Abnormalities: Update of a 2006 Review-Comparative Effectiveness Review No. 47. Rockville, MD: Agency for Healthcare Research and Quality; February 2012. AHRQ publication 12-EHC014-EF.

3. Gershon-Cohen J. The importance of $\mathrm{x}$-ray microcalcifications in breast cancer. AJR. 1967;99:1010-1011.

4. Baker R, Rogers KD, Shepard N, Stone N. New relationships between breast microcalcifications and cancer. Br J Cancer. 2010;103:1034-1039.

5. Going JJ, Anderson TJ, Crocker PR, Levison DA. Weddellite calcification in the breast: eighteen cases with implications for breast cancer screening. Histopathology. 1990;16:119-124.

6. Haka AS, Shafer-Peltier KE, Fitzmaurice M, Crowe J, Dasari RR, Feld MS. Identifying microcalcifications in benign and malignant breast lesions by probing differences in their chemical composition using Raman spectroscopy. Cancer Res. 2002;62:5375-5380.

7. Morgan MP, Cooke MM, Christopherson PA, Westfall PR, McCarthy GM. Calcium hydroxyapatite promotes mitogenesis and matrix metalloproteinase expression in human breast cancer cell lines. Mol Carcinog. 2001;32:111-117.

8. Cox RF, Hernandez-Santana A, Ramdass S, McMahon G, Harmey JH, Morgan MP. Microcalcifications in breast cancer: novel insights into the molecular mechanism and functional consequence of mammary mineralisation. $\mathrm{Br} \mathrm{J}$ Cancer. 2012;106:525-537.

9. Pleshko N, Boskey A, Mendelsohn R. Novel infrared spectroscopic method for the determination of crystallinity of hydroxyapatite minerals. Biophys J. 1991;60:786-793.

10. Nelson DG. The influence of carbonate on the atomic structure and reactivity of hydroxyapatite. J Dent Res. 1981;60 spec no C:1621-1629.

11. Hawkins RA, Hoh C, Glaspy J, et al. The role of positron emission tomography in oncology and other whole-body applications. Semin Nucl Med. 1992;22:268-284.

12. Czernin J, Satyamurthy N, Schiepers C. Molecular mechanisms of bone ${ }^{18} \mathrm{~F}-\mathrm{NaF}$ deposition. J Nucl Med. 2010;51:1826-1829.

13. Korah RM, Sysounthone V, Golowa Y, Wieder R. Basic fibroblast growth factor confers a less malignant phenotype in MDA-MB-231 human breast cancer cells. Cancer Res. 2000;60:733-740.

14. Aboagye EO, Bhujwalla ZM. Malignant transformation alters membrane choline phospholipid metabolism of human mammary epithelial cells. Cancer Res. 1999; 59:80-84.

15. Uddin MJ, Crews BC, Ghebreselasie K, et al. Fluorinated COX-2 inhibitors as agents in PET imaging of inflammation and cancer. Cancer Prev Res (Phila). 2011;4:1536-1545. 
16. Nelson DG, Featherstone JD. Preparation, analysis, and characterization of carbonated apatites. Calcif Tissue Int. 1982;34(suppl 2):S69-S81.

17. Loening AM, Gambhir SS. AMIDE: a free software tool for multimodality medical image analysis. Mol Imaging. 2003;2:131-137.

18. Blau M, Ganatra R, Bender MA. 18 F-fluoride for bone imaging. Semin Nucl Med. 1972;2:31-37.

19. Schmid HA, Requintina PJ, Oxenkrug GF, Sturner W. Calcium, calcification, and melatonin biosynthesis in the human pineal gland: a postmortem study into agerelated factors. J Pineal Res. 1994;16:178-183.

20. Krstić R, Golaz J. Ultrastructural and x-ray microprobe comparison of gerbil and human pineal acervuli. Experientia. 1977;33:507-508.

21. Alcolado JC, Moore IE, Weller RO. Calcification in the human choroid plexus, meningiomas and pineal gland. Neuropathol Appl Neurobiol. 1986;12:235-250.

22. Coe FL, Parks JH, Asplin JR. The pathogenesis and treatment of kidney stones. N Engl J Med. 1992;327:1141-1152.

23. Lemann J Jr. Composition of the diet and calcium kidney stones. $N$ Engl J Med. 1993;328:880-882.

24. Parmar MS. Kidney stones. BMJ. 2004;328:1420-1424.

25. Moe OW. Kidney stones: pathophysiology and medical management. Lancet. 2006;367:333-344.
26. Low RK, Ho S, Stoller ML. Sodium fluoride dissolution of human calcium oxalate/phosphate stone particles. J Endourol. 1995;9:379-382.

27. Cook GJ. Oncological molecular imaging: nuclear medicine techniques. $\mathrm{Br} \mathrm{J}$ Radiol. 2003;76(spec no 2):S152-S158.

28. Segall G, Delbeke D, Stabin MG, et al. SNM practice guideline for sodium ${ }^{18} \mathrm{~F}$ fluoride PET/CT bone scans 1.0. J Nucl Med. 2010;51:1813-1820.

29. Wiechmann L, Kuerer HM. The molecular journey from ductal carcinoma in situ to invasive breast cancer. Cancer. 2008;112:2130-2142.

30. Moran M, Haffty BG. Lobular carcinoma in situ as a component of breast cancer: the long-term outcome in patients treated with breast-conservation therapy. Int J Radiat Oncol Biol Phys. 1998;40:353-358.

31. Aulmann S, Penzel R, Schirmacher P, Sinn HP. Lobular carcinoma in situ (LCIS): risk factor and precursor of invasive lobular breast cancer [in German]. Verh Dtsch Ges Pathol. 2007;91:208-213.

32. Iagaru A, Mittra E, Yaghoubi SS, et al. Novel strategy for a cocktail ${ }^{18} \mathrm{~F}$-fluoride and ${ }^{18}$ F-FDG PET/CT scan for evaluation of malignancy: results of the pilotphase study. J Nucl Med. 2009;50:501-505.

33. Doot RK, Muzi M, Peterson LM, et al. Kinetic analysis of ${ }^{18} \mathrm{~F}$-fluoride PET images of breast cancer bone metastases. J Nucl Med. 2010;51: $521-527$. 